\title{
A new class of travelling wave solutions for local fractional diffusion differential equations
}

\author{
Ziyue Shi ${ }^{1}$, Wei Qi ${ }^{2 *}$ and Jing Fan ${ }^{1}$
}

"Correspondence:
youaaaa@163.com
2Department of Physics, China
University of Mining and
Technology, Xuzhou, China
Full list of author information is
available at the end of the article

\begin{abstract}
In this paper, we investigate a 3-D diffusion equation within the scope of the local fractional derivative. For this model, we establish local fractional vector operators and a local fractional Laplace operator defined on Cantor-type cylindrical coordinate and Cantor-type spherical coordinate, respectively. With the help of the spherical symmetry method based on those operators, we obtain exact traveling wave solutions of the 3-D diffusion equation. The results reveal that the proposed schemes are very effective for obtaining nondifferentiable solutions of fractional diffusion problems.
\end{abstract}

Keywords: Time-space fractional diffusion equation; Local fractional derivative; Cantor-type cylindrical coordinate; Traveling wave

\section{Introduction}

In recent years, classical mathematics has been greatly enriched because of the advancement and application of fractional calculus. Numerous researches have shown that the models with fractional calculous are able to describe the reality more accurately than the classical models with integer-order derivatives $[4,13]$. Consequently, fractional calculus has been widely employed in the mathematical modeling of issues in various branches of science [2,3] and engineering [18]. Various fractional calculus operators, such as Riemann-Liouville, Caputo, and Atangana-Baleanu calculus [25], are introduced and studied in a wide variety of literature. Many approaches, such as the Laplace and Fourier transform methods, are introduced to get analytical solutions of fractional-order differential equations. However, the fractional-order differential equations derived from the real world are often nonlinear and extremely complex, and most of them cannot get accurate analytic solutions. As a result, the Adomian decomposition method [1], homotopy analysis transform method [16], and fractional Laplace decomposition technique [17] are used to get numerical solutions of the models.

Although most fractional calculus operators have good properties of describing dynamic systems, they also have some limitations. For example, they can only deal with differentiable problems. Recently, a new approach, local fractional calculus (LFC) (a kind of fractal

(c) The Author(s) 2020. This article is licensed under a Creative Commons Attribution 4.0 International License, which permits use sharing, adaptation, distribution and reproduction in any medium or format, as long as you give appropriate credit to the original author(s) and the source, provide a link to the Creative Commons licence, and indicate if changes were made. The images or other third party material in this article are included in the article's Creative Commons licence, unless indicated otherwise in a credit line to the material. If material is not included in the article's Creative Commons licence and your intended use is not permitted by statutory regulation or exceeds the permitted use, you will need to obtain permission directly from the copyright holder. To view a copy of this licence, visit http://creativecommons.org/licenses/by/4.0/. 
calculus), which was originally proposed by Kolwankar and Gangal [5, 6], was presented to resolve nondifferentiable problems in a fractal time-space, and its important properties were developed. In recent years, local fractional calculus has become increasingly popular and gained important advancement due mainly to its outstanding properties in modeling complex nonlinear dynamical systems in different branches of mathematical physics, such as nanoscale flows [19], heat transmission [36], diffusion on Cantor sets [34], and others. Several analytical methods, such as the local fractional homotopy perturbation Sumudu transform method [26], local fractional variational iteration algorithm [37] and local fractional Fourier series [33], have been proposed to address local fractional partial differential equations.

There has been a growing interest in the fractional diffusion phenomenon [14, 20, 22] that describes changes in the net moment concentration of substances such as solids, liquids, gases, and so on [15]. Fractional calculus theory was widely employed to describe the diffusion phenomenon in engineering, and fractional diffusion equations were discussed in the literature [7, 11, 21, 23, 24, 27, 28, 31].

In this paper, we consider the 3-D diffusion equation defined on Cantor sets with local fractional derivative given by

$$
\frac{\partial^{\alpha} u(x, y, z, t)}{\partial t^{\alpha}}=\mu\left[\frac{\partial^{2 \alpha} u(x, y, z, t)}{\partial x^{2 \alpha}}+\frac{\partial^{2 \alpha} u(x, y, z, t)}{\partial y^{2 \alpha}}+\frac{\partial^{2 \alpha} u(x, y, z, t)}{\partial z^{2 \alpha}}\right],
$$

where $\alpha(0<\alpha<1)$ is a fractal dimension, $\mu$ is a constant, and $u(x, y, z, t)$ is the local diffusion model with respect to space $(x, y, z)$ and time $t$.

The main our objective is determining the exact traveling wave solution for the fractional diffusion (Eq. (1.1)) in 3-D Cantorian coordinate systems. The problem has not been solved for the time being, and the authors try to take inspiration from the theory of integer-order differential equations.

As we know, the spherical symmetry method in the differential equation of integral order can be described as follows:

$$
\frac{\partial u}{\partial t}=a^{2}\left(\frac{\partial^{2} u}{\partial x^{2}}+\frac{\partial^{2} u}{\partial y^{2}}+\frac{\partial^{2} u}{\partial z^{2}}\right)
$$

where $a$ is a constant, and $u(x, y, z, t)$ is a function with respect to space $(x, y, z)$ and time $t$. If $u(x, y, z, t)$ is spherically symmetric with respect to $(x, y, z)$,

$$
u(x, y, z, t)=u(r, t), r=\sqrt{x^{2}+y^{2}+z^{2}},
$$

we find that Eq. (1.2) can be rewritten in the form

$$
\frac{\partial u}{\partial t}=a^{2}\left(\frac{\partial^{2} u}{\partial r^{2}}+\frac{2}{r} \frac{\partial u}{\partial r}\right)
$$

Thus we obtain from Eq. (1.4) the 1-D differential equation of integer order

$$
\frac{\partial(r u)}{\partial t}=a^{2}\left(\frac{\partial^{2}(r u)}{\partial r^{2}}\right)
$$


Motivated by these ideas, we try to reduce the 3-D diffusion equation with local fractional differential operator into a 1-D one utilizing the method of spherical symmetry of fractional order, which is similar to the spherical symmetry method of integer order. However, we cannot get a satisfactory result by using the Cantor-type spherical coordinate method, which was set up on fractals first constructed in [32].

In this paper, we derive local fractional vector operators and the Laplace operator in the Cantor-type cylindrical coordinates and Cantor-type spherical coordinates according to the theories of local fractional calculus. The results have some differences from those presented in [32]. The advantage of the method is that it helps degrade the dimension of the 3-D diffusion equation (1.1), which is a key step in seeking for the exact traveling wave solution for the equation. Finally, we present the graph of the traveling wave solution of the diffusion equation.

The structure of this paper is as follows. In Sect. 2, we present basic theories of local fractional derivatives (LFDs) and local fractional integral (LFI). In Sect. 3, we reduce the transformations of vector operators and he Laplace operator between Cartesian coordinate system and Cantor-type cylindrical or spherical coordinates, respectively. Then we prpose the symmetric Cantor-type cylindrical and symmetric spherical coordinates. In Sect. 4, we investigate a new class of traveling wave solutions for the 3-D fractional diffusion equation (1.1) defined on Cantor sets. Then we give an example of an initial value problem of a 3-D fractional diffusion equation to demonstrate the proposed methods.

\section{Preliminaries}

In this section, we introduce the basic definitions and properties about local fractional calculus [35].

Let $C_{\alpha}(a, b)$ be a set of nondifferentiable functions (NFs).

Let $f \in C_{\alpha}(a, b)$. The local fractional derivative of an NF $f$ of order $\alpha(0<\alpha<1)$ at the point $x=x_{0}$ is defined by

$$
D^{(\alpha)} f\left(x_{0}\right)=\left.\frac{d^{\alpha} f(x)}{d x^{\alpha}}\right|_{x=x_{0}}=\lim _{x \rightarrow x_{0}} \frac{\Delta^{\alpha}\left[f(x)-f\left(x_{0}\right)\right]}{\left(x-x_{0}\right)^{\alpha}}
$$

where $\Delta^{\alpha}\left[f(x)-f\left(x_{0}\right)\right] \cong \Gamma(1+\alpha)\left[f(x)-f\left(x_{0}\right)\right]$ with the Euler gamma function $\Gamma(1+\alpha):=$ $\int_{0}^{\infty} \mu^{\alpha-1} \exp (-\mu) d \mu$.

Suppose that $D^{(\alpha)} f\left(x_{0}\right)$ exist for all $x_{0} \in(a, b)$. In this case, $D_{\alpha}(a, b)$ is called the local fractional derivative set.

Let $f, g \in D_{\alpha}(a, b)$. The properties of the LFDs are as follows:

$$
\begin{aligned}
& D^{(\alpha)}[f(x) \pm g(x)]=D^{(\alpha)} f(x) \pm D^{(\alpha)} g(x), \\
& D^{(\alpha)}[f(x) g(x)]=g(x) D^{(\alpha)} f(x)+f(x) D^{(\alpha)} g(x), \\
& D^{(\alpha)}[f(x) / g(x)]=\left[D^{(\alpha)} f(x)\right] g(x)-\left[D^{(\alpha)} g(x)\right] f(x) / g^{2}(x),
\end{aligned}
$$

provided that $g(x) \neq 0$. 
Suppose that $f(x)=(\phi \circ \varphi)(x), x \in(a, b)$. The local fractional chain rule via the interchanging operator of nondifferential functions are as follows:

$$
\frac{d^{\alpha} f(x)}{d x^{\alpha}}=\phi^{(\alpha)}(\varphi)\left[\varphi^{(1)}(x)\right]^{\alpha}
$$

if $\phi^{(\alpha)}(\varphi)$ and $\varphi^{(1)}(x)$ exist.

The generalized functions defined on Cantor sets are given by

$$
E_{\alpha}\left(x^{\alpha}\right)=\sum_{n=0}^{\infty} \frac{x^{n \alpha}}{\Gamma(1+n \alpha)} .
$$

The sine and cosine functions on a fractal set are given by

$$
\begin{aligned}
& \sin _{\alpha}\left(x^{\alpha}\right)=\frac{E_{\alpha}\left(i^{\alpha} x^{\alpha}\right)-E_{\alpha}\left(-i^{\alpha} x^{\alpha}\right)}{2 i^{\alpha}}=\sum_{k=0}^{\infty} \frac{(-1)^{k} \mu^{(2 k+1) \alpha}}{\Gamma(1+(2 k+1) \alpha)}, \\
& \cos _{\alpha}\left(x^{\alpha}\right)=\frac{E_{\alpha}\left(i^{\alpha} x^{\alpha}\right)+E_{\alpha}\left(-i^{\alpha} x^{\alpha}\right)}{2}=\sum_{k=0}^{\infty} \frac{(-1)^{k} \mu^{2 k \alpha}}{\Gamma(1+2 k \alpha)},
\end{aligned}
$$

where $\mu \in R, 0<\alpha<1$, and $i^{\alpha}$ is a imaginary unit of a fractal set.

There are several formulas of the LFDs of NFs:

$$
\begin{aligned}
& D^{(\alpha)}\left[x^{n \alpha} / \Gamma(1+n \alpha)\right]=x^{(n-1) \alpha} / \Gamma(1+(n-1) \alpha), \\
& D^{(\alpha)}\left[E_{\alpha}\left(x^{\alpha}\right)\right]=E_{\alpha}\left(x^{\alpha}\right) \\
& D^{(\alpha)}\left[E_{\alpha}\left(C x^{\alpha}\right)\right]=C E_{\alpha}\left(C x^{\alpha}\right) \\
& D^{(\alpha)}\left[E_{\alpha}\left(x^{2 \alpha}\right)\right]=(2 x)^{\alpha} E_{\alpha}\left(x^{2 \alpha}\right) \\
& D^{(\alpha)}\left[\sin _{\alpha}\left(x^{\alpha}\right)\right]=\cos _{\alpha}\left(x^{\alpha}\right) \\
& D^{(\alpha)}\left[\cos _{\alpha}\left(x^{\alpha}\right)\right]=-\sin _{\alpha}\left(x^{\alpha}\right) \\
& D^{(\alpha)}\left[\sin _{\alpha}^{\alpha}\left(x^{\alpha}\right)\right]=\Gamma(1+\alpha) \cos _{\alpha}\left(x^{\alpha}\right) \\
& D^{(\alpha)}\left[\cos _{\alpha}^{\alpha}\left(x^{\alpha}\right)\right]=-\Gamma(1+\alpha) \sin _{\alpha}\left(x^{\alpha}\right)
\end{aligned}
$$

Let $f \in C_{\alpha}(a, b)$. The LFI of the NF $f(x)$ in the interval $[a, b]$ is defined by

$$
\begin{aligned}
{ }_{a} I_{b}^{(\alpha)} f(x) & =\frac{1}{\Gamma(1+\alpha)} \int_{a}^{b} f(x)(d x)^{\alpha} \\
& =\frac{1}{\Gamma(1+\alpha)} \lim _{\Delta x \rightarrow 0} \sum_{i=0}^{i=N-1} f(x)(\Delta x)^{\alpha},
\end{aligned}
$$

where $\Delta x=x_{i+1}-x_{i}, i=0,1, \ldots, N-1, x_{0}=a$, and $x_{N}=b$.

The properties of the LFI are as follows:

$$
\begin{aligned}
& \frac{1}{\Gamma(1+\alpha)} \int_{a}^{x}\left[D^{(\alpha)} f(x)\right](d t)^{\alpha}=f(x)-f(a), \\
& D^{(\alpha)}\left[\frac{1}{\Gamma(1+\alpha)} \int_{a}^{x} \Phi(t)(d t)^{\alpha}\right]=\Phi(x) .
\end{aligned}
$$




\section{Local fractional operators in Cantor-type cylindrical and spherical coordinates}

Certain shapes, like circular or spherical ones, are more easily determined in cylindrical or spherical coordinates than in Cartesian coordinates. Both classical and fractional differential equations in the coordinate system are switched between Cartesian, cylindrical, and spherical coordinates [8]. In this section, we describe the Cantor-type cylindrical coordinate method and Cantor-type spherical coordinate method to investigate a family of local fractional differential operators on Cantor sets, such as the local fractional gradient operator, local fractional divergence operator, and local fractional Laplace operator.

\subsection{The Cantor-type cylindrical coordinate system}

Let us consider the coordinate system of the Cantor-type cylindrical coordinates

$$
\begin{aligned}
\mathbf{r} & =x^{\alpha} \mathbf{e}_{1}^{\alpha}+y^{\alpha} \mathbf{e}_{2}^{\alpha}+z^{\alpha} \mathbf{e}_{3}^{\alpha} \\
& =r_{R} \mathbf{e}_{R}^{\alpha}+r_{\theta} \mathbf{e}_{\theta}^{\alpha}+r_{z} \mathbf{e}_{z}^{\alpha},
\end{aligned}
$$

where $0<\alpha<1, e_{i}^{\alpha}, i=1,2,3$, are the directions of the local fractional vector functions,

$$
\begin{aligned}
& r_{R}=r(R, \theta, z) \cdot \mathbf{e}_{R}^{\alpha}, \\
& r_{\theta}=r(R, \theta, z) \cdot \mathbf{e}_{\theta}^{\alpha}, \\
& r_{z}=r(R, \theta, z) \cdot \mathbf{e}_{z}^{\alpha}, \\
& r(R, \theta, z)=r \cdot\left(\mathbf{e}_{R}^{\alpha}+\mathbf{e}_{\theta}^{\alpha}+\mathbf{e}_{z}^{\alpha}\right), \\
& r=R^{\alpha} \cos _{\alpha}\left(\theta^{\alpha}\right) \mathbf{e}_{1}^{\alpha}+R^{\alpha} \sin _{\alpha}\left(\theta^{\alpha}\right) \mathbf{e}_{2}^{\alpha}+z^{\alpha} \mathbf{e}_{3}^{\alpha},
\end{aligned}
$$

and

$$
\left\{\begin{array}{l}
x^{\alpha}=R^{\alpha} \cos _{\alpha}\left(\theta^{\alpha}\right), \\
y^{\alpha}=R^{\alpha} \sin _{\alpha}\left(\theta^{\alpha}\right), \\
z^{\alpha}=z^{\alpha},
\end{array}\right.
$$

with $R \in(0,+\infty), \theta \in(0,2 \pi), z \in(-\infty,+\infty)$, and $x^{2 \alpha}+y^{2 \alpha}=R^{2 \alpha}$.

In this case, we have that

$$
\begin{aligned}
\frac{\partial^{\alpha}}{\partial R^{\alpha}} & =\left(\frac{\partial x}{\partial R}\right)^{\alpha} \frac{\partial^{\alpha}}{\partial x^{\alpha}}+\left(\frac{\partial y}{\partial R}\right)^{\alpha} \frac{\partial^{\alpha}}{\partial y^{\alpha}}+\left(\frac{\partial z}{\partial R}\right)^{\alpha} \frac{\partial^{\alpha}}{\partial z^{\alpha}} \\
& =\mathbf{e}_{R}^{\alpha} \cdot \nabla^{\alpha} \\
& =\nabla_{R}^{\alpha}, \\
\frac{\partial^{\alpha}}{\partial \theta^{\alpha}} & =\left(\frac{\partial x}{\partial \theta}\right)^{\alpha} \frac{\partial^{\alpha}}{\partial x^{\alpha}}+\left(\frac{\partial y}{\partial \theta}\right)^{\alpha} \frac{\partial^{\alpha}}{\partial y^{\alpha}}+\left(\frac{\partial z}{\partial \theta}\right)^{\alpha} \frac{\partial^{\alpha}}{\partial z^{\alpha}} \\
& =R^{\alpha} \mathbf{e}_{\theta}^{\alpha} \cdot \nabla^{\alpha} \\
& =R^{\alpha} \nabla_{\theta}^{\alpha},
\end{aligned}
$$




$$
\begin{aligned}
\frac{\partial^{\alpha}}{\partial z^{\alpha}} & =\left(\frac{\partial x}{\partial z}\right)^{\alpha} \frac{\partial^{\alpha}}{\partial x^{\alpha}}+\left(\frac{\partial y}{\partial z}\right)^{\alpha} \frac{\partial^{\alpha}}{\partial y^{\alpha}}+\left(\frac{\partial z}{\partial z}\right)^{\alpha} \frac{\partial^{\alpha}}{\partial z^{\alpha}} \\
& =\mathbf{e}_{z}^{\alpha} \cdot \nabla^{\alpha} \\
& =\nabla_{z}^{\alpha} .
\end{aligned}
$$

We have

$$
\left\{\begin{array}{l}
\nabla_{R}^{\alpha}=\mathbf{e}_{R}^{\alpha} \cdot \nabla^{\alpha}=\frac{\partial^{\alpha}}{\partial R^{\alpha}}, \\
\nabla_{\theta}^{\alpha}=\mathbf{e}_{\theta}^{\alpha} \cdot \nabla^{\alpha}=\frac{1}{R^{\alpha}} \frac{\partial^{\alpha}}{\partial \theta^{\alpha}}, \\
\nabla_{z}^{\alpha}=\mathbf{e}_{z}^{\alpha} \cdot \nabla^{\alpha}=\frac{\partial^{\alpha}}{\partial z^{\alpha}},
\end{array}\right.
$$

where

$$
\begin{aligned}
\mathbf{e}_{R}^{\alpha} & =\left(\left(\frac{\partial x}{\partial R}\right)^{\alpha},\left(\frac{\partial y}{\partial R}\right)^{\alpha},\left(\frac{\partial z}{\partial R}\right)^{\alpha}\right) \\
& =\left(\cos _{\alpha}^{\alpha}\left(\theta^{\alpha}\right), \sin _{\alpha}^{\alpha}\left(\theta^{\alpha}\right), 0\right), \\
\mathbf{e}_{\theta}^{\alpha} & =\left(\left(\frac{\partial x}{\partial R}\right)^{\alpha},\left(\frac{\partial y}{\partial R}\right)^{\alpha},\left(\frac{\partial z}{\partial R}\right)^{\alpha}\right) \\
& =\left(-\sin _{\alpha}^{\alpha}\left(\theta^{\alpha}\right), \cos _{\alpha}^{\alpha}\left(\theta^{\alpha}\right), 0\right), \\
\mathbf{e}_{z}^{\alpha} & =\left(\left(\frac{\partial x}{\partial z}\right)^{\alpha},\left(\frac{\partial y}{\partial z}\right)^{\alpha},\left(\frac{\partial z}{\partial z}\right)^{\alpha}\right) \\
& =(0,0,1), \\
\nabla^{\alpha} & =\left(\frac{\partial^{\alpha}}{\partial x^{\alpha}}, \frac{\partial^{\alpha}}{\partial y^{\alpha}}, \frac{\partial^{\alpha}}{\partial z^{\alpha}}\right) .
\end{aligned}
$$

The local fractional gradient operator in the Cantor-type cylindrical coordinates reads as

$$
\begin{aligned}
\nabla^{\alpha} u & =\left(\mathbf{e}_{R}^{\alpha} \nabla_{R}^{\alpha} u, \mathbf{e}_{\theta}^{\alpha} \nabla_{\theta}^{\alpha} u, \mathbf{e}_{z}^{\alpha} \nabla_{z}^{\alpha} u\right) \\
& =\left(\mathbf{e}_{R}^{\alpha} \frac{\partial^{\alpha} u}{\partial R^{\alpha}}, \mathbf{e}_{\theta}^{\alpha} \frac{1}{R^{\alpha}} \frac{\partial^{\alpha} u}{\partial \theta^{\alpha}}, \mathbf{e}_{z}^{\alpha} \frac{\partial^{\alpha} u}{\partial z^{\alpha}}\right)
\end{aligned}
$$

We obtain the local fractional divergence operator in the Cantor-type cylindrical coordinates

$$
\begin{aligned}
\nabla^{\alpha} \cdot \mathbf{r} & =\mathbf{e}_{R}^{\alpha} \cdot \frac{\partial^{\alpha} \mathbf{r}}{\partial R^{\alpha}}+\mathbf{e}_{\theta}^{\alpha} \cdot \frac{1}{R^{\alpha}} \frac{\partial^{\alpha} \mathbf{r}}{\partial \theta^{\alpha}}+\mathbf{e}_{z}^{\alpha} \cdot \frac{\partial^{\alpha} \mathbf{r}}{\partial z^{\alpha}} \\
& =\frac{\partial^{\alpha} r_{R}}{\partial R^{\alpha}}+\frac{\Gamma(1+\alpha) r_{R}}{R^{\alpha}}+\frac{1}{R^{\alpha}} \frac{\partial^{\alpha} r_{\theta}}{\partial \theta^{\alpha}}+\frac{\partial^{\alpha} r_{z}}{\partial z^{\alpha}} .
\end{aligned}
$$

We get the local fractional Laplace operator in the Cantor-type cylindrical coordinates:

$$
\nabla^{2 \alpha} u=\left(\mathbf{e}_{R}^{\alpha} \frac{\partial^{\alpha}}{\partial R^{\alpha}}+\mathbf{e}_{\theta}^{\alpha} \frac{1}{R^{\alpha}} \frac{\partial^{\alpha}}{\partial \theta^{\alpha}}+\mathbf{e}_{z}^{\alpha} \frac{\partial^{\alpha}}{\partial z^{\alpha}}\right) \cdot\left(\mathbf{e}_{R}^{\alpha} \frac{\partial^{\alpha} u}{\partial R^{\alpha}}+\mathbf{e}_{\theta}^{\alpha} \frac{1}{R^{\alpha}} \frac{\partial^{\alpha} u}{\partial \theta^{\alpha}}+\mathbf{e}_{z}^{\alpha} \frac{\partial^{\alpha} u}{\partial z^{\alpha}}\right)
$$


where

$$
\begin{aligned}
& \mathbf{e}_{R}^{\alpha} \cdot \frac{\partial^{\alpha}}{\partial R^{\alpha}}\left(\mathbf{e}_{R}^{\alpha} \frac{\partial^{\alpha} u}{\partial R^{\alpha}}+\mathbf{e}_{\theta}^{\alpha} \frac{1}{R^{\alpha}} \frac{\partial^{\alpha} u}{\partial \theta^{\alpha}}+\mathbf{e}_{z}^{\alpha} \frac{\partial^{\alpha} u}{\partial z^{\alpha}}\right) \\
& =\mathbf{e}_{R}^{\alpha} \cdot\left(\frac{\partial^{\alpha} u}{\partial R^{\alpha}} \frac{\partial^{\alpha} \mathbf{e}_{R}^{\alpha}}{\partial R^{\alpha}}+\mathbf{e}_{R}^{\alpha} \frac{\partial^{\alpha}}{\partial R^{\alpha}}\left(\frac{\partial^{\alpha} u}{\partial R^{\alpha}}\right)+\frac{1}{R^{\alpha}} \frac{\partial^{\alpha} u}{\partial \theta^{\alpha}} \frac{\partial^{\alpha} \mathbf{e}_{\theta}^{\alpha}}{\partial R^{\alpha}}+\mathbf{e}_{\theta}^{\alpha} \frac{\partial^{\alpha}}{\partial R^{\alpha}}\left(\frac{1}{R^{\alpha}} \frac{\partial^{\alpha} u}{\partial \theta^{\alpha}}\right)\right. \\
& \left.+\frac{\partial^{\alpha} u}{\partial z^{\alpha}} \frac{\partial^{\alpha} \mathbf{e}_{z}^{\alpha}}{\partial R^{\alpha}}+\mathbf{e}_{z}^{\alpha} \frac{\partial^{\alpha}}{\partial R^{\alpha}}\left(\frac{\partial^{\alpha} u}{\partial z^{\alpha}}\right)\right) \\
& =\frac{\partial^{2 \alpha} u}{\partial R^{2 \alpha}} \\
& \mathbf{e}_{\theta}^{\alpha} \cdot \frac{1}{R^{\alpha}} \frac{\partial^{\alpha}}{\partial \theta^{\alpha}}\left(\mathbf{e}_{R}^{\alpha} \frac{\partial^{\alpha} u}{\partial R^{\alpha}}+\mathbf{e}_{\theta}^{\alpha} \frac{1}{R^{\alpha}} \frac{\partial^{\alpha} u}{\partial \theta^{\alpha}}+\mathbf{e}_{z}^{\alpha} \frac{\partial^{\alpha} u}{\partial z^{\alpha}}\right) \\
& =\frac{1}{R^{\alpha}} \mathbf{e}_{\theta}^{\alpha} \cdot\left(\frac{\partial^{\alpha} u}{\partial R^{\alpha}} \frac{\partial^{\alpha} \mathbf{e}_{R}^{\alpha}}{\partial \theta^{\alpha}}+\mathbf{e}_{R}^{\alpha} \frac{\partial^{\alpha}}{\partial \theta^{\alpha}}\left(\frac{\partial^{\alpha} u}{\partial R^{\alpha}}\right)+\frac{1}{R^{\alpha}} \frac{\partial^{\alpha} u}{\partial \theta^{\alpha}} \frac{\partial^{\alpha} \mathbf{e}_{\theta}^{\alpha}}{\partial \theta^{\alpha}}+\mathbf{e}_{\theta}^{\alpha} \frac{\partial^{\alpha}}{\partial \theta^{\alpha}}\left(\frac{1}{R^{\alpha}} \frac{\partial^{\alpha} u}{\partial \theta^{\alpha}}\right)\right. \\
& \left.+\frac{\partial^{\alpha} u}{\partial z^{\alpha}} \frac{\partial^{\alpha} \mathbf{e}_{z}^{\alpha}}{\partial \theta^{\alpha}}+\mathbf{e}_{z}^{\alpha} \frac{\partial^{\alpha}}{\partial \theta^{\alpha}}\left(\frac{\partial^{\alpha} u}{\partial z^{\alpha}}\right)\right) \\
& =\frac{1}{R^{\alpha}} \mathbf{e}_{\theta}^{\alpha} \cdot \frac{\partial^{\alpha} u}{\partial R^{\alpha}} \frac{\partial^{\alpha} \mathbf{e}_{R}^{\alpha}}{\partial \theta^{\alpha}}+\frac{1}{R^{2 \alpha}} \frac{\partial^{2 \alpha} u}{\partial \theta^{2 \alpha}} \\
& =\frac{1}{R^{\alpha}} \frac{\partial^{\alpha} u}{\partial R^{\alpha}} \mathbf{e}_{\theta}^{\alpha} \cdot\left(\frac{\partial^{\alpha} \cos _{\alpha}^{\alpha}\left(\theta^{\alpha}\right)}{\partial \theta^{\alpha}}, \frac{\partial^{\alpha} \sin _{\alpha}^{\alpha}\left(\theta^{\alpha}\right)}{\partial \theta^{\alpha}}, \frac{\partial^{\alpha} 0}{\partial \theta^{\alpha}}\right)+\frac{1}{R^{2 \alpha}} \frac{\partial^{2 \alpha} u}{\partial \theta^{2 \alpha}} \\
& =\frac{1}{R^{\alpha}} \frac{\partial^{\alpha} u}{\partial R^{\alpha}} \mathbf{e}_{\theta}^{\alpha} \cdot\left(-\Gamma(1+\alpha) \sin _{\alpha}^{\alpha}\left(\theta^{\alpha}\right), \Gamma(1+\alpha) \cos ^{\alpha}\left(\theta^{\alpha}\right), 0\right)+\frac{1}{R^{2 \alpha}} \frac{\partial^{2 \alpha} u}{\partial \theta^{2 \alpha}} \\
& =\frac{\Gamma(1+\alpha)}{R^{\alpha}} \frac{\partial^{\alpha} u}{\partial R^{\alpha}}+\frac{1}{R^{2 \alpha}} \frac{\partial^{2 \alpha} u}{\partial \theta^{2 \alpha}}, \\
& \mathbf{e}_{z}^{\alpha} \cdot \frac{\partial^{\alpha}}{\partial z^{\alpha}}\left(\mathbf{e}_{R}^{\alpha} \frac{\partial^{\alpha} u}{\partial R^{\alpha}}+\mathbf{e}_{\theta}^{\alpha} \frac{1}{R^{\alpha}} \frac{\partial^{\alpha} u}{\partial \theta^{\alpha}}+\mathbf{e}_{z}^{\alpha} \frac{\partial^{\alpha} u}{\partial z^{\alpha}}\right) \\
& =\mathbf{e}_{z}^{\alpha} \cdot\left(\frac{\partial^{\alpha} u}{\partial R^{\alpha}} \frac{\partial^{\alpha} \mathbf{e}_{R}^{\alpha}}{\partial z^{\alpha}}+\mathbf{e}_{R}^{\alpha} \frac{\partial^{\alpha}}{\partial z^{\alpha}}\left(\frac{\partial^{\alpha} u}{\partial R^{\alpha}}\right)+\frac{1}{R^{\alpha}} \frac{\partial^{\alpha} u}{\partial \theta^{\alpha}} \frac{\partial^{\alpha} \mathbf{e}_{\theta}^{\alpha}}{\partial z^{\alpha}}+\mathbf{e}_{\theta}^{\alpha} \frac{\partial^{\alpha}}{\partial z^{\alpha}}\left(\frac{1}{R^{\alpha}} \frac{\partial^{\alpha} u}{\partial \theta^{\alpha}}\right)\right. \\
& \left.+\frac{\partial^{\alpha} u}{\partial z^{\alpha}} \frac{\partial^{\alpha} \mathbf{e}_{z}^{\alpha}}{\partial z^{\alpha}}+\mathbf{e}_{z}^{\alpha} \frac{\partial^{\alpha}}{\partial z^{\alpha}}\left(\frac{\partial^{\alpha} u}{\partial z^{\alpha}}\right)\right) \\
& =\frac{\partial^{2 \alpha} u}{\partial z^{2 \alpha}} \text {. }
\end{aligned}
$$

Hence the local fractional Laplace operator in the Cantor-type cylindrical coordinates can be written in the form

$$
\nabla^{2 \alpha} u=\frac{\partial^{2 \alpha} u}{\partial R^{2 \alpha}}+\frac{\Gamma(1+\alpha)}{R^{\alpha}} \frac{\partial^{\alpha} u}{\partial R^{\alpha}}+\frac{1}{R^{2 \alpha}} \frac{\partial^{2 \alpha} u}{\partial \theta^{2 \alpha}}+\frac{\partial^{2 \alpha} u}{\partial z^{2 \alpha}} .
$$

\subsection{The Cantor-type spherical coordinate system}

We consider the coordinate system of the Cantor-type spherical coordinates

$$
\begin{aligned}
\mathbf{r} & =x^{\alpha} \mathbf{e}_{1}^{\alpha}+y^{\alpha} \mathbf{e}_{2}^{\alpha}+z^{\alpha} \mathbf{e}_{3}^{\alpha} \\
& =r_{R} \mathbf{e}_{R}^{\alpha}+r_{\theta} \mathbf{e}_{\theta}^{\alpha}+r_{\varphi} \mathbf{e}_{\varphi}^{\alpha},
\end{aligned}
$$


where

$$
\left\{\begin{array}{l}
x^{\alpha}=R^{\alpha} \sin _{\alpha}\left(\theta^{\alpha}\right) \cos _{\alpha}\left(\varphi^{\alpha}\right), \\
y^{\alpha}=R^{\alpha} \sin _{\alpha}\left(\theta^{\alpha}\right) \sin _{\alpha}\left(\varphi^{\alpha}\right), \\
z^{\alpha}=R^{\alpha} \cos _{\alpha}\left(\theta^{\alpha}\right)
\end{array}\right.
$$

with $R \in(0,+\infty), \theta \in(0, \pi), \varphi \in(0,2 \pi)$, and $x^{2 \alpha}+y^{2 \alpha}+z^{2 \alpha}=R^{2 \alpha}$.

In this case, we have that

$$
\begin{aligned}
\frac{\partial^{\alpha}}{\partial R^{\alpha}} & =\left(\frac{\partial x}{\partial R}\right)^{\alpha} \frac{\partial^{\alpha}}{\partial x^{\alpha}}+\left(\frac{\partial y}{\partial R}\right)^{\alpha} \frac{\partial^{\alpha}}{\partial y^{\alpha}}+\left(\frac{\partial z}{\partial R}\right)^{\alpha} \frac{\partial^{\alpha}}{\partial z^{\alpha}} \\
& =\mathbf{e}_{R}^{\alpha} \cdot \nabla^{\alpha} \\
& =\nabla_{R}^{\alpha}, \\
\frac{\partial^{\alpha}}{\partial \theta^{\alpha}} & =\left(\frac{\partial x}{\partial \theta}\right)^{\alpha} \frac{\partial^{\alpha}}{\partial x^{\alpha}}+\left(\frac{\partial y}{\partial \theta}\right)^{\alpha} \frac{\partial^{\alpha}}{\partial y^{\alpha}}+\left(\frac{\partial z}{\partial \theta}\right)^{\alpha} \frac{\partial^{\alpha}}{\partial z^{\alpha}} \\
& =R^{\alpha} \mathbf{e}_{\theta}^{\alpha} \cdot \nabla^{\alpha} \\
& =R^{\alpha} \nabla_{\theta}^{\alpha}, \\
\frac{\partial^{\alpha}}{\partial \varphi^{\alpha}} & =\left(\frac{\partial x}{\partial \varphi}\right)^{\alpha} \frac{\partial^{\alpha}}{\partial x^{\alpha}}+\left(\frac{\partial y}{\partial \varphi}\right)^{\alpha} \frac{\partial^{\alpha}}{\partial y^{\alpha}}+\left(\frac{\partial z}{\partial \varphi}\right)^{\alpha} \frac{\partial^{\alpha}}{\partial z^{\alpha}} \\
& =R^{\alpha} \sin _{\alpha}^{\alpha}\left(\theta^{\alpha}\right) \mathbf{e}_{\varphi}^{\alpha} \cdot \nabla^{\alpha} \\
& =R^{\alpha} \sin _{\alpha}^{\alpha}\left(\theta^{\alpha}\right) \nabla_{\varphi}^{\alpha} .
\end{aligned}
$$

We have

$$
\left\{\begin{aligned}
\nabla_{R}^{\alpha} & =\mathbf{e}_{R}^{\alpha} \cdot \nabla^{\alpha}=\frac{\partial^{\alpha}}{\partial R^{\alpha}}, \\
\nabla_{\theta}^{\alpha} & =\mathbf{e}_{\theta}^{\alpha} \cdot \nabla^{\alpha}=\frac{1}{R^{\alpha}} \frac{\partial^{\alpha}}{\partial \theta^{\alpha}}, \\
\nabla_{\varphi}^{\alpha} & =\mathbf{e}_{\varphi}^{\alpha} \cdot \nabla^{\alpha}=\frac{1}{R^{\alpha} \sin _{\alpha}^{\alpha}\left(\theta^{\alpha}\right)} \frac{\partial^{\alpha}}{\partial \varphi^{\alpha}},
\end{aligned}\right.
$$

where

$$
\begin{aligned}
\mathbf{e}_{R}^{\alpha} & =\left(\left(\frac{\partial x}{\partial R}\right)^{\alpha},\left(\frac{\partial y}{\partial R}\right)^{\alpha},\left(\frac{\partial z}{\partial R}\right)^{\alpha}\right) \\
& =\left(\sin _{\alpha}^{\alpha}\left(\theta^{\alpha}\right) \cos _{\alpha}^{\alpha}\left(\varphi^{\alpha}\right), \sin _{\alpha}^{\alpha}\left(\theta^{\alpha}\right) \sin _{\alpha}^{\alpha}\left(\varphi^{\alpha}\right), \cos _{\alpha}^{\alpha}\left(\theta^{\alpha}\right)\right), \\
\mathbf{e}_{\theta}^{\alpha} & =\frac{1}{R^{\alpha}}\left(\left(\frac{\partial x}{\partial \theta}\right)^{\alpha},\left(\frac{\partial y}{\partial \theta}\right)^{\alpha},\left(\frac{\partial z}{\partial \theta}\right)^{\alpha}\right) \\
& =\left(\cos _{\alpha}^{\alpha}\left(\theta^{\alpha}\right) \cos _{\alpha}^{\alpha}\left(\varphi^{\alpha}\right), \cos _{\alpha}^{\alpha}\left(\theta^{\alpha}\right) \sin _{\alpha}^{\alpha}\left(\varphi^{\alpha}\right),-\sin _{\alpha}^{\alpha}\left(\theta^{\alpha}\right)\right), \\
\mathbf{e}_{\varphi}^{\alpha} & =\frac{1}{R^{\alpha} \sin _{\alpha}^{\alpha}\left(\theta^{\alpha}\right)}\left(\left(\frac{\partial x}{\partial \varphi}\right)^{\alpha},\left(\frac{\partial y}{\partial \varphi}\right)^{\alpha},\left(\frac{\partial z}{\partial \varphi}\right)^{\alpha}\right) \\
& =\left(-\sin _{\alpha}^{\alpha}\left(\varphi^{\alpha}\right), \cos _{\alpha}^{\alpha}\left(\varphi^{\alpha}\right), 0\right), \\
\nabla^{\alpha} & =\left(\frac{\partial^{\alpha}}{\partial x^{\alpha}}, \frac{\partial^{\alpha}}{\partial y^{\alpha}}, \frac{\partial^{\alpha}}{\partial z^{\alpha}}\right) .
\end{aligned}
$$


The local fractional gradient operator in the Cantor-type spherical coordinates is written as follows:

$$
\begin{aligned}
\nabla^{\alpha} u & =\left(\mathbf{e}_{R}^{\alpha} \nabla_{R}^{\alpha} u, \mathbf{e}_{\theta}^{\alpha} \nabla_{\theta}^{\alpha} u, \mathbf{e}_{\varphi}^{\alpha} \nabla_{\varphi}^{\alpha} u\right) \\
& =\left(\mathbf{e}_{R}^{\alpha} \frac{\partial^{\alpha} u}{\partial R^{\alpha}}, \mathbf{e}_{\theta}^{\alpha} \frac{1}{R^{\alpha}} \frac{\partial^{\alpha} u}{\partial \theta^{\alpha}}, \mathbf{e}_{\varphi}^{\alpha} \frac{1}{R^{\alpha} \sin _{\alpha}^{\alpha}\left(\theta^{\alpha}\right)} \frac{\partial^{\alpha} u}{\partial \varphi^{\alpha}}\right) .
\end{aligned}
$$

Hence we get the local fractional divergence operator in the Cantor-type spherical coordinates

$$
\begin{aligned}
\nabla^{\alpha} \cdot \mathbf{r}= & \mathbf{e}_{R}^{\alpha} \cdot \frac{\partial^{\alpha} \mathbf{r}}{\partial R^{\alpha}}+\mathbf{e}_{\theta}^{\alpha} \cdot \frac{1}{R^{\alpha}} \frac{\partial^{\alpha} \mathbf{r}}{\partial \theta^{\alpha}}+\mathbf{e}_{\varphi}^{\alpha} \cdot \frac{1}{R^{\alpha} \sin _{\alpha}^{\alpha}\left(\theta^{\alpha}\right)} \frac{\partial^{\alpha} \mathbf{r}}{\partial \varphi^{\alpha}} \\
= & \frac{\partial^{\alpha} r_{R}}{\partial R^{\alpha}}+\frac{2 \Gamma(1+\alpha) r_{R}}{R^{\alpha}}+\frac{1}{R^{\alpha}} \frac{\partial^{\alpha} r_{\theta}}{\partial \theta^{\alpha}} \\
& +\frac{1}{R^{\alpha} \sin _{\alpha}^{\alpha}\left(\theta^{\alpha}\right)}\left(\frac{\partial^{\alpha} r_{\varphi}}{\partial \varphi^{\alpha}}+\Gamma(1+\alpha) r_{\theta} \cos _{\alpha}^{\alpha}\left(\theta^{\alpha}\right)\right) .
\end{aligned}
$$

The local fractional Laplace operator in the Cantor-type spherical coordinates is presented as follows:

$$
\begin{aligned}
\nabla^{2 \alpha} u= & \left(\mathbf{e}_{R}^{\alpha} \frac{\partial^{\alpha}}{\partial R^{\alpha}}+\mathbf{e}_{\theta}^{\alpha} \frac{1}{R^{\alpha}} \frac{\partial^{\alpha}}{\partial \theta^{\alpha}}+\mathbf{e}_{\varphi}^{\alpha} \frac{1}{R^{\alpha} \sin _{\alpha}^{\alpha}\left(\theta^{\alpha}\right)} \frac{\partial^{\alpha}}{\partial \varphi^{\alpha}}\right) \\
& \cdot\left(\mathbf{e}_{R}^{\alpha} \frac{\partial^{\alpha} u}{\partial R^{\alpha}}+\mathbf{e}_{\theta}^{\alpha} \frac{1}{R^{\alpha}} \frac{\partial^{\alpha} u}{\partial \theta^{\alpha}}+\mathbf{e}_{\varphi}^{\alpha} \frac{1}{R^{\alpha} \sin _{\alpha}^{\alpha}\left(\theta^{\alpha}\right)} \frac{\partial^{\alpha} u}{\partial \varphi^{\alpha}}\right),
\end{aligned}
$$

where

$$
\begin{aligned}
& \mathbf{e}_{R}^{\alpha} \cdot \frac{\partial^{\alpha}}{\partial R^{\alpha}}\left(\mathbf{e}_{R}^{\alpha} \frac{\partial^{\alpha} u}{\partial R^{\alpha}}+\mathbf{e}_{\theta}^{\alpha} \frac{1}{R^{\alpha}} \frac{\partial^{\alpha} u}{\partial \theta^{\alpha}}+\mathbf{e}_{\varphi} \frac{1}{R^{\alpha} \sin _{\alpha}^{\alpha}\left(\theta^{\alpha}\right)} \frac{\partial^{\alpha} u}{\partial \varphi^{\alpha}}\right)=\frac{\partial^{2 \alpha} u}{\partial R^{2 \alpha}}, \\
& \mathbf{e}_{\theta}^{\alpha} \cdot \frac{1}{R^{\alpha}} \frac{\partial^{\alpha}}{\partial \theta^{\alpha}}\left(\mathbf{e}_{R}^{\alpha} \frac{\partial^{\alpha} u}{\partial R^{\alpha}}+\mathbf{e}_{\theta}^{\alpha} \frac{1}{R^{\alpha}} \frac{\partial^{\alpha} u}{\partial \theta^{\alpha}}+\mathbf{e}_{\varphi}^{\alpha} \frac{1}{R^{\alpha} \sin _{\alpha}^{\alpha}\left(\theta^{\alpha}\right)} \frac{\partial^{\alpha} u}{\partial \varphi^{\alpha}}\right) \\
& =\frac{1}{R^{2 \alpha}} \frac{\partial^{2 \alpha} u}{\partial \theta^{2 \alpha}}+\frac{\Gamma(1+\alpha)}{R^{\alpha}} \frac{\partial^{\alpha} u}{\partial R^{\alpha}}, \\
& \mathbf{e}_{\varphi}^{\alpha} \frac{1}{R^{\alpha} \sin _{\alpha}^{\alpha}\left(\theta^{\alpha}\right)} \cdot \frac{\partial^{\alpha}}{\partial \varphi^{\alpha}}\left(\mathbf{e}_{R}^{\alpha} \frac{\partial^{\alpha} u}{\partial R^{\alpha}}+\mathbf{e}_{\theta}^{\alpha} \frac{1}{R^{\alpha}} \frac{\partial^{\alpha} u}{\partial \theta^{\alpha}}+\mathbf{e}_{\varphi}^{\alpha} \frac{1}{R^{\alpha} \sin _{\alpha}^{\alpha}\left(\theta^{\alpha}\right)} \frac{\partial^{\alpha} u}{\partial \varphi^{\alpha}}\right) \\
& \quad=\frac{\Gamma(1+\alpha)}{R^{\alpha}} \frac{\partial^{\alpha} u}{\partial R^{\alpha}}+\frac{\Gamma(1+\alpha)}{R^{2 \alpha}} \frac{\cos _{\alpha}^{\alpha}\left(\theta^{\alpha}\right)}{\sin _{\alpha}^{\alpha}\left(\theta^{\alpha}\right)} \frac{\partial^{\alpha} u}{\partial \theta^{\alpha}}+\frac{1}{R^{2 \alpha} \sin _{\alpha}^{\alpha}\left(\theta^{\alpha}\right)} \frac{\partial^{2 \alpha} u}{\partial \varphi^{2 \alpha}} .
\end{aligned}
$$

Thus the local fractional Laplace operator in the Cantor-type spherical coordinates takes the form

$$
\begin{aligned}
\nabla^{2 \alpha} u= & \frac{\partial^{2 \alpha} u}{\partial R^{2 \alpha}}+\frac{2 \Gamma(1+\alpha)}{R^{\alpha}} \frac{\partial^{\alpha} u}{\partial R^{\alpha}}+\frac{1}{R^{2 \alpha}} \frac{\partial^{2 \alpha} u}{\partial \theta^{2 \alpha}}+\frac{\Gamma(1+\alpha)}{R^{2 \alpha}} \frac{\cos _{\alpha}^{\alpha}\left(\theta^{\alpha}\right)}{\sin _{\alpha}^{\alpha}\left(\theta^{\alpha}\right)} \frac{\partial^{\alpha} u}{\partial \theta^{\alpha}} \\
& +\frac{1}{R^{2 \alpha} \sin _{\alpha}^{\alpha}\left(\theta^{\alpha}\right)} \frac{\partial^{2 \alpha} u}{\partial \varphi^{2 \alpha}} .
\end{aligned}
$$

Remark 3.1 We have the Cantor-type cylindrical and spherical symmetries as follows: 
Adopting Eq. (3.17), the Cantor-type cylindrical symmetry form of local fractional Laplace operator is presented as

$$
\nabla^{2 \alpha} u=\frac{\partial^{2 \alpha} u}{\partial R^{2 \alpha}}+\frac{\Gamma(1+\alpha)}{R^{\alpha}} \frac{\partial^{\alpha} u}{\partial R^{\alpha}} .
$$

Adopting Eq. (3.34), the Cantor-type spherical symmetry form of the local fractional Laplace operator is presented as

$$
\nabla^{2 \alpha} u=\frac{\partial^{2 \alpha} u}{\partial R^{2 \alpha}}+\frac{2 \Gamma(1+\alpha)}{R^{\alpha}} \frac{\partial^{\alpha} u}{\partial R^{\alpha}} .
$$

\section{Exact traveling wave solution of nondifferentiability}

Fractional diffusion theories have stimulated a growing interest and importance in complex systems due mainly to its widespread applications in numerous kinds of materials in nature, such as turbid material [12], superconducting alloys [29], and lattice [30]. In this section, we investigate the exact traveling wave solutions for the 3-D fractional diffusion equation (1.1) defined on Cantor sets with local fractional differential operator.

In view of Eq. (3.34), we change Eq. (1.1) into the 3-D fractional diffusion equation in the Cantor-type spherical coordinate system

$$
\begin{aligned}
\frac{d^{\alpha} u}{d t^{\alpha}}= & \mu\left[\frac{\partial^{2 \alpha} u}{\partial R^{2 \alpha}}+\frac{2 \Gamma(1+\alpha)}{R^{\alpha}} \frac{\partial^{\alpha} u}{\partial R^{\alpha}}+\frac{1}{R^{2 \alpha}} \frac{\partial^{2 \alpha} u}{\partial \theta^{2 \alpha}}+\frac{\Gamma(1+\alpha)}{R^{2 \alpha}} \frac{\cos _{\alpha}^{\alpha}\left(\theta^{\alpha}\right)}{\sin _{\alpha}^{\alpha}\left(\theta^{\alpha}\right)} \frac{\partial^{\alpha} u}{\partial \theta^{\alpha}}\right. \\
& \left.+\frac{1}{R^{2 \alpha} \sin _{\alpha}^{\alpha}\left(\theta^{\alpha}\right)} \frac{\partial^{2 \alpha} u}{\partial \varphi^{2 \alpha}}\right] .
\end{aligned}
$$

Using Eq. (3.36), the Cantor-type spherical symmetry form of the diffusion reads

$$
\frac{d^{\alpha} u}{d t^{\alpha}}=\mu\left[\frac{\partial^{2 \alpha} u}{\partial R^{2 \alpha}}+\frac{2 \Gamma(1+\alpha)}{R^{\alpha}} \frac{\partial^{\alpha} u}{\partial R^{\alpha}}\right]
$$

which is rewritten in the form

$$
R^{\alpha} \frac{d^{\alpha} u}{d t^{\alpha}}=\mu\left[R^{\alpha} \frac{\partial^{2 \alpha} u}{\partial R^{2 \alpha}}+2 \Gamma(1+\alpha) \frac{\partial^{\alpha} u}{\partial R^{\alpha}}\right]
$$

Due to the chain rule of the LFD (see Eq. (2.5)), Eq. (4.3) becomes

$$
\frac{\partial^{\alpha}\left(R^{\alpha} u\right)}{\partial t^{\alpha}}=\mu \frac{\partial^{2 \alpha}\left(R^{\alpha} u\right)}{\partial R^{2 \alpha}} .
$$

Take

$$
\Omega(R, t)=R^{\alpha} u(x, y, z, t),
$$

where $R^{2 \alpha}=x^{2 \alpha}+y^{2 \alpha}+z^{2 \alpha}$.

Thus we obtain from Eq. (4.4) the local fractional diffusion model, which is nondifferentable in time $t$ and space $R$ :

$$
\frac{\partial^{\alpha} \Omega}{\partial t^{\alpha}}=\mu \frac{\partial^{2 \alpha} \Omega}{\partial R^{2 \alpha}} .
$$


Therefore the solution of the local fractional 3-D diffusion equation (1.1) can be written as

$$
u(x, y, z, t)=\frac{1}{R^{\alpha}} \Omega(R, t)
$$

where $R^{2 \alpha}=x^{2 \alpha}+y^{2 \alpha}+z^{2 \alpha}$.

To find the exact traveling wave solutions of Eq. (1.1), the diffusion model Eq. (4.6) should be solved first. We consider

$$
\varphi^{\alpha}=R^{\alpha}-v^{\alpha} t^{\alpha}
$$

where

$$
\lim _{\alpha \rightarrow 1}=R-v t
$$

with the wave speed $v$.

Due to the chain rule of the LFD (see Eq. (2.5)), we have

$$
\begin{aligned}
& \frac{\partial^{\alpha} \Omega}{\partial t^{\alpha}}=\frac{\partial^{\alpha} \Omega}{\partial \varphi^{\alpha}}\left(\frac{\partial \varphi}{\partial t}\right)^{\alpha}=-v^{\alpha} \frac{\partial^{\alpha} \Omega}{\partial \varphi^{\alpha}}, \\
& \frac{\partial^{\alpha} \Omega}{\partial R^{\alpha}}=\frac{\partial^{\alpha} \Omega}{\partial \varphi^{\alpha}}\left(\frac{\partial \varphi}{\partial R}\right)^{\alpha}=\frac{\partial^{\alpha} \Omega}{\partial \varphi^{\alpha}}, \\
& \frac{\partial^{2 \alpha} \Omega}{\partial R^{2 \alpha}}=\frac{\partial^{2 \alpha} \Omega}{\partial \varphi^{2 \alpha}},
\end{aligned}
$$

where $v^{\alpha}$ is the fractal wave speed.

Substituting Eqs. (4.10)-(4.12) into Eq. (4.6) gives the local fractional diffusion model

$$
v^{\alpha} \frac{\partial^{\alpha} \Omega}{\partial \varphi^{\alpha}}+\mu \frac{\partial^{2 \alpha} \Omega}{\partial \varphi^{2 \alpha}}=0
$$

which is rewritten in the form

$$
\frac{v^{\alpha}}{\mu} \frac{\partial^{\alpha} \Omega}{\partial \varphi^{\alpha}}+\frac{\partial^{2 \alpha} \Omega}{\partial \varphi^{2 \alpha}}=0 .
$$

From Eq. (4.14) we obtain

$$
\frac{\partial^{\alpha}\left(\frac{v^{\alpha}}{\mu} \Omega\right)}{\partial \varphi^{\alpha}}+\frac{\partial^{\alpha}}{\partial \varphi^{\alpha}}\left(\frac{\partial^{\alpha} \Omega}{\partial \varphi^{\alpha}}\right)=0 .
$$

Finding the LFI of Eq. (4.15) with respect to $\varphi$ yields

$$
\frac{v^{\alpha}}{\mu} \Omega+\frac{\partial^{\alpha} \Omega}{\partial \varphi^{\alpha}}=C_{1},
$$

where $C_{1}$ is a constant. 
Taking $C_{1}=0$, from Eq. (4.16) we obtain

$$
\frac{\nu^{\alpha}}{\mu} \Omega+\frac{\partial^{\alpha} \Omega}{\partial \varphi^{\alpha}}=0
$$

Thus we easily obtain Eq. (4.17) when the LFI of Eq. (4.15) yields a term that decays. To find the exact traveling wave solution of nondifferentiable type of Eq. (4.6), we derive

$$
\frac{\partial^{\alpha}\left[a E_{\alpha}\left(b \varphi^{\alpha}\right)\right]}{\partial \varphi^{\alpha}}=a b E_{\alpha}\left(b \varphi^{\alpha}\right)=b\left[a E_{\alpha}\left(b \varphi^{\alpha}\right)\right] .
$$

In view of Eq. (4.18), we have

$$
\Omega=a E_{\alpha}\left(b \varphi^{\alpha}\right)
$$

such that

$$
\frac{\partial^{\alpha} \Omega}{\partial \varphi^{\alpha}}-b \Omega=0
$$

Taking $a=1$ and $b=-\frac{v^{\alpha}}{\mu}$, we have

$$
\Omega(\varphi)=E_{\alpha}\left(-\frac{\nu^{\alpha}}{\mu} \varphi^{\alpha}\right) .
$$

Thus, in view of Eq. (4.8), the exact traveling wave solution of Eq. (4.6) takes the form

$$
\Omega(R, t)=E_{\alpha}\left[-\frac{v^{\alpha}}{\mu}\left(R^{\alpha}-v^{\alpha} t^{\alpha}\right)\right] .
$$

Therefore the exact traveling wave solution of nondifferentiable type of Eq. (1.1) can be written as

$$
u(x, y, z, t)=\frac{1}{R^{\alpha}} E_{\alpha}\left[-\frac{v^{\alpha}}{\mu}\left(R^{\alpha}-v^{\alpha} t^{\alpha}\right)\right]
$$

where $R^{2 \alpha}=x^{2 \alpha}+y^{2 \alpha}+z^{2 \alpha}$.

Remark 4.1 We consider the following initial value of Eq. (1.1):

$$
u(R, 0)=\frac{1}{R^{\alpha}} E_{\alpha}\left(R^{\alpha}\right)
$$

where $R^{2 \alpha}=x^{2 \alpha}+y^{2 \alpha}+z^{2 \alpha}$.

By Eq. (4.23) we have

$$
u(R, 0)=\frac{1}{R^{\alpha}} E_{\alpha}\left(-\frac{v^{\alpha}}{\mu} R^{\alpha}\right) .
$$

Taking

$$
R^{\alpha}=-\frac{v^{\alpha}}{\mu} R^{\alpha}
$$




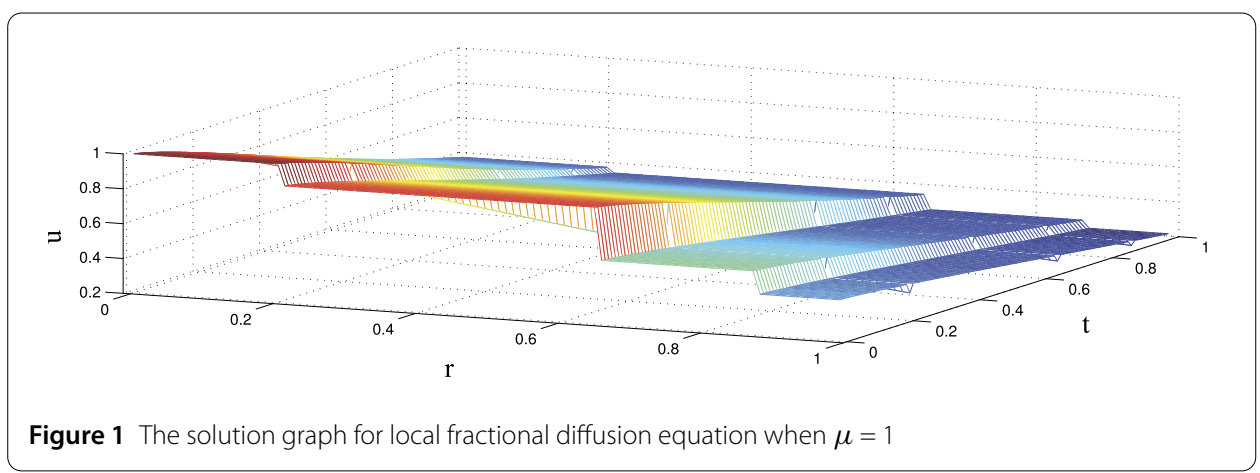

we have

$$
v^{\alpha}=-\mu \text {. }
$$

Therefore we obtain the exact traveling wave solution of the fractional diffusion equation

$$
u(x, y, z, t)=\frac{1}{R^{\alpha}} E_{\alpha}\left(R^{\alpha}+\mu t^{\alpha}\right),
$$

where $R^{2 \alpha}=x^{2 \alpha}+y^{2 \alpha}+z^{2 \alpha}$.

In Fig. 1, we show the graph of the solution for the local fractional diffusion equation when $\mu=1$.

\section{Conclusion}

In this paper, we investigated a 3-D fractional diffusion equation defined on Cantor sets with local fractional derivative, which is quite useful in solving nondifferentiable problems in fractal time-space. We have derived transformations of local fractional vector operators and the local fractional Laplace operator in the Cantor-type cylindrical and spherical coordinates, and those operators are little different from the existing research results. In addition, the operators in Cantor-type coordinates are slightly different from those in Cartesian-type coordinates, but they are unified when the fractal dimension $\alpha=1$. Then the exact non-differentiable traveling wave solutions for a 3-D diffusion equation with local fractional derivative were obtained by the spherical symmetry method, which depends on the local fractional differential operator transformations derived above. To have a better understanding of the method, we discussed the traveling wave solution for the initial value problem of a 3-D fractional diffusion equation. The result indicates that the suggested schemes are very simple and computationally attractive to handle similar kinds of differential equations. As a future research direction of this paper, the new methods derived in this manuscript can be extended to get analytical solutions of other models presented in other works published recently associated with high-dimensional fractional equations $[9,10]$.

Acknowledgements

The authors thank the referees for useful comments.

Funding

This work is supported by the National Nature Science Foundation of China grant (11501560). 
Availability of data and materials

Data sharing not applicable to this paper as no data was generated or analyzed during the current study.

\section{Competing interests}

The authors declare that they have no competing interests.

\section{Authors' contributions}

The authors contributed equally to the manuscript and typed, read, and approved the final manuscript.

\section{Author details}

'Department of Mathematics, China University of Mining and Technology, Xuzhou, China. ${ }^{2}$ Department of Physics, China University of Mining and Technology, Xuzhou, China.

\section{Publisher's Note}

Springer Nature remains neutral with regard to jurisdictional claims in published maps and institutional affiliations.

Received: 25 August 2019 Accepted: 8 January 2020 Published online: 28 February 2020

\section{References}

1. Adomian, G.: Solving Frontier Problems of Physics: The Decomposition Method. Kluwer Academic, Boston (1999)

2. Baleanu, D., Asad, J.H., Jajarmi, A.: New aspects of the motion of a particle in a circular cavity. Proc. Rom. Acad., Ser. A $19(2), 361-367$ (2018)

3. Baleanu, D., Jajarmi, A., Sajjadi, S.S., Mozyrska, D.: A new fractional model and optimal control of a tumor-immune surveillance with non-singular derivative operator. Chaos 2019, Article ID 083127 (2019)

4. Baleanu, D., Sajjadi, S.S., Jajarmi, A., Asad, J.H.: New features of the fractional Euler-Lagrange equations for a physical system within non-singular derivative operator. Eur. Phys. J. Plus 134(4), 181 (2019)

5. Carpinteri, A., Chiaia, B., Cornetti, P.: Static-kinematic duality and the principle of virtual work in the mechanics of fractal media. Comput. Methods Appl. Math. 191(1), 3-19 (2001)

6. Carpinteri, A., Chiaia, B., Cornetti, P.: The elastic problem for fractal media: basic theory and finite element formulation. Comput. Struct. 82(6), 499-508 (2004)

7. Chaves, A.S.: A fractional diffusion equation to describe Lévy flights. Phys. Lett. A 239(1-2), 13-16 (1998)

8. Ganji, Z.Z., Ganji, D.D., Ganji, A.D.G., Rostamian, M.: Analytical solution of time-fractional Navier-Stokes equation in polar coordinate by homotopy perturbation method. Numer. Methods Partial Differ. Equ. 26(1), 117-124 (2010)

9. Hajipour, M., Jajarmi, A., Baleanu, D.: On the accurate discretization of a highly nonlinear boundary value problem. Numer. Algorithms 79(3), 679-695 (2018)

10. Hajipour, M., Jajarmi, A., Malek, A., Baleanu, D.: Positivity-preserving sixth-order implicit finite difference weighted essentially non-oscillatory scheme for the nonlinear heat equation. Appl. Math. Comput. 325, 146-158 (2018)

11. Hristov, J.: Approximate solutions to fractional subdiffusion equations. Eur. Phys. J. Spec. Top. 193(1), 229-243 (2011)

12. Ishimaru, A.: Diffusion of light in turbid material. Appl. Opt. 28(12), 2210-2215 (1989)

13. Jajarmi, A., Arshad, S., Baleanu, D.: A new fractional modelling and control strategy for the outbreak of dengue fever. Physica A 535, Article ID 122524 (2019)

14. Jesus, I.S., Tenreiro Machado, J.A.: Fractional control of heat diffusion system. Nonlinear Dyn. 54(3), $263-282$ (2008)

15. Jost, W.: Diffusion in Solids, Liquids, Gases. Academic Press, New York (1952)

16. Kumar, D., Singh, J., Baleanu, D., Rathore, S.: Analysis of a fractional model of the Ambartsumian equation. Eur. Phys. J. Plus 133(7), 259 (2018)

17. Kumar, D., Singh, J., Tanwar, K., Baleanu, D.: A new fractional exothermic reactions model having constant heat source in porous media with power, exponential and Mittag-Leffler laws. Int. J. Heat Mass Transf. 138, 1222-1227 (2019)

18. Kumar, D., Tchier, F., Singh, J., Baleanu, D.: An efficient computational technique for fractal vehicular traffic flow. Entropy 20(4), 259 (2018)

19. Liu, H.Y., He, J.H., Li, Z.B.: Fractional calculus for nanoscale flow and heat transfer. Int. J. Numer. Methods Heat Fluid Flow 24(6), 1227-1250 (2014)

20. Luchko, Y.: Maximum principle for the generalized time-fractional diffusion equation. J. Math. Anal. Appl. 351(1) 218-223 (2009)

21. Mainardi, F., Pagnini, G.: The Wright functions as solutions of the time-fractional diffusion equation. Appl. Math. Comput. 141(1), 51-62 (2003)

22. Mainardi, F., Pagnini, G., Gorenflo, R.: Some aspects of fractional diffusion equations of single and distributed order. Appl. Math. Comput. 187(1), 295-305 (2007)

23. Povstenko, Y.: Time-fractional radial diffusion in a sphere. Nonlinear Dyn. 53(53), 55-65 (2007)

24. Povstenko, Y.: Fractional radial diffusion in an infinite medium with a cylindrical cavity. Q. Appl. Math. 67(1), 113-123 (2009)

25. Singh, J., Kumar, D., Baleanu, D.: New aspects of fractional Biswas-Milovic model with Mittag-Leffler law. Math. Model. Nat. Phenom. 14(3), 303 (2019)

26. Singh, J., Kumar, D., Baleanu, D., Rathore, S.: An efficient numerical algorithm for the fractional Drinfeld-Sokolov-Wilson equation. Appl. Math. Comput. 335, 12-24 (2018)

27. Sokolov, I.M., Chechkin, A.V., Klafter, J.: Fractional diffusion equation for a power-law-truncated Lévy process. Physica A 336(3-4), 245-251 (2004)

28. Tadjerana, C.: A second-order accurate numerical approximation for the fractional diffusion equation. J. Comput. Phys. 213(1), 205-213 (2006)

29. Usadel, K.D.: Generalized diffusion equation for superconducting alloys. Phys. Rev. Lett. 25(8), 507-509 (1970)

30. Wolf-Gladrow, D.: A lattice Boltzmann equation for diffusion. J. Stat. Phys. 79(5-6), 1023-1032 (1995)

31. Wyss, W.: The fractional diffusion equation. J. Math. Phys. 27(11), 2782-2785 (1986) 
32. Yang, X.J.: Local Fractional Functional Analysis and Its Applications. Asian Academic, Hong Kong (2011)

33. Yang, X.J.: Advanced Local Fractional Calculus and Its Applications. World Science, New York (2012)

34. Yang, X.J., Baleanu, D., Srivastava, H.M.: Local fractional similarity solution for the diffusion equation defined on Cantor sets. Appl. Math. Lett. 47, 54-60 (2015)

35. Yang, X.J., Baleanu, D., Srivastava, H.M.: Local Fractional Integral Transforms and Their Applications. Academic Press, New York (2015)

36. Yang, X.J., Srivastava, H.M., He, J.H., Baleanu, D.: Cantor-type cylindrical-coordinate method for differential equations with local fractional derivatives. Phys. Lett. A 377(28-30), 1696-1700 (2013)

37. Zhang, Y., Srivastava, H.M., Baleanu, M.C.: Local fractional variational iteration algorithm II for non-homogeneous model associated with the non-differentiable heat flow. Adv. Mech. Eng. (2015). https://doi.org/10.1177/1687814015608567

Submit your manuscript to a SpringerOpen ${ }^{\circ}$ journal and benefit from:

- Convenient online submission

- Rigorous peer review

- Open access: articles freely available online

- High visibility within the field

- Retaining the copyright to your article

Submit your next manuscript at $\boldsymbol{\nabla}$ springeropen.com 